\title{
Characteristics of dogs having diabetes mellitus; analysis of data from private Japanese veterinary clinics
}

Shiho Usui', Hidemi Yasuda ${ }^{2}$ and Yuzo Koketsu ${ }^{1 *}$

*Correspondence: koket001@isc.meiji.ac.jp

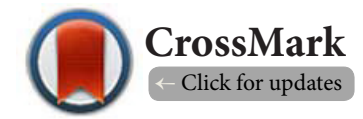

'School of Agriculture, Meiji University, Kawasaki, Japan.

${ }^{2}$ Spectrum Lab Japan, Midorigaoka, Meguro-ku, Tokyo, Japan.

\begin{abstract}
Our objective was to characterize dogs having diabetes mellitus by age, sex, neuter status, body condition score, and body size or breeds. The dataset comprised medical records of 5,343 diabetic or non-diabetic dogs from 102 breeds collected from 1,198 clinics between 2006 and 2013. Dogs were categorized into four body condition score groups. Multilevel logistic regression models were applied to the data. Dogs having diabetes mellitus were characterized by high age, underweight and thin dogs, and also body size or specific breeds such as Toy Poodles $(\mathrm{P}<0.05)$, but there was no association with sex $(\mathrm{P}=0.41)$ or neuter status $(\mathrm{P}=0.68)$. The odds ratio of dogs having DM was 5.6 for underweight and thin dogs compared with ideal dogs $(\mathrm{P}<0.05)$. The probability of underweight and thin dogs having diabetes mellitus increased by $16.7 \%$ from 1 to 7 years old $(\mathrm{P}<0.05)$. The odds ratios of Toy Poodles and Miniature Schnauzers were 3.1 and 1.6, respectively $(\mathrm{P}<0.05)$ compared to Other breeds. Additionally, the probability of Toy Poodles having diabetes mellitus increased by $16.4 \%$ from 5 to 12 years old $(\mathrm{P}<0.05)$, whereas in Shetland Sheepdogs it only increased by $0.4 \%$ over the same age range. In conclusion, veterinarians need to consider age, body condition and breed when identifying subclinical diabetic dogs.
\end{abstract}

Keywords: Canine, characteristics, diabetes mellitus, descriptive epidemiology

\section{Introduction}

Diabetes mellitus (DM) is one of the most frequently diagnosed endocrine diseases in dogs, accounting for $0.32-0.64 \%$ of dogs receiving veterinary care in U.K. and U.S.A. [1-3]. Dogs with DM have been diagnosed by polydipsia, polyuria, polyphagia and weight loss [4]. The cause of canine DM is considered multifactorial, i.e., autoimmune destruction of insulin producing $\beta$ cells, pancreatitis, chronic hyperglycemia or hormonal disturbances [4]. Also, middle aged to older dogs and intact female dogs have been associated with dog DM [2]. Additionally, excessive overweight in dogs causes hyperinsulinemia and glucose intolerance [2]. Other studies have shown that Miniature Schnauzers and Toy Poodles are high risk breeds for DM [5], whereas Shetland Sheepdogs and Golden Retrievers have reduced risk of DM [1].

No studies in Japan have quantified the characteristic factors (age, sex, neuter status, body condition and breeds) associated with DM in dogs, nor the interactions between these factors in the same model, using medical records. Also, dogs' veterinary medical records are in a multi-level structure because health related checks, guidance and treatments on an individual dog are all performed in a clinic, i.e., dogs are not independent of the clinic. The clinic is a variable that includes some unique information, such as a clinic's location, the average social and economic status of all owners coming with their dogs to the clinic and veterinary health guidance. Therefore, the objective of the present study was to characterize DM in dogs by using a mixed-effects modelling approach to identify characteristic factors and estimate their effects.

\section{Materials and methods}

Database and body condition score (BCS)

A dog database has been created at Meiji University (Kawasaki, Japan) by cooperating with a veterinary service (Spectrum Lab Japan, Tokyo, Japan). The veterinary service recorded information about individual dog's characteristics (age, sex, neuter status, BCS and breed) when they received serum samples for lipoprotein analysis from veterinarians in private clinics 
throughout Japan. The veterinarians who submitted the samples were not informed about the specific purposes of the present study. The serum samples were collected from clinically-non-diseased dogs that received a health check and from dogs that were being assessed for suspected dyslipidemia or DM. Dogs' health condition was diagnosed by their veterinarians when the serum samples were taken. The diagnosis of DM was determined by the veterinarians on the basis of DM criteria such as polydipsia and polyuria with blood glucose and urine glycosuria [4]. However, the veterinarians did not record separately whether the dogs had insulin deficiency DM or insulin resistance DM. The BCS for each dog was evaluated by the dog's veterinarian using a five-point scale system (1: thin, 2: underweight, 3: ideal, 4: overweight and 5: obese). In addition to the five-point BCS scale used in our study, there is also a nine-point scale system [6] that has been commonly used in the EU and the U.S.A. However, we used the five-point scale system [7] because the website information and brochures about the five-point BCS scale system are widely available to veterinary clinics across Japan, provided by Pet Food Institute (Washington D.C., U.S.A.) and a nutrition company (Hill's-Colgate JAPAN Ltd., Tokyo, Japan). At the time of the study, three most popular types of dogs in Japan were Toy Poodle, Chihuahua and Miniature Dachshund [8].

\section{Data and exclusion criteria}

The database comprised data of 7,982 dogs from 109 breeds, collected from 1,198 veterinary clinics across Japan between January 2006 and January 2013. Of the 11,032 small animal clinics in Japan [9], 10.9\% were included in our project. Records of second visits or more were not used in the present study $(1,886$ records). Records of dogs having hypothyroidism or hyperadrenocorticism health problems, which would influence body condition, were excluded from the dataset (753 records). The diagnoses of these endocrine diseases were made by the dogs' veterinarians on the basis of symptoms such as polydipsia and polyuria with blood and urinary tests. Thus, the dataset included the records of 5,343 diabetic or non-diabetic dogs in 102 breeds from 1,131 clinics.

The proportions of the 6,096 samples included in East Japan (including Tokyo) and West Japan were 61.7 and 38.3\%, respectively. The samples were submitted from all the 47 prefecture regions in Japan. Additionally, the proportions of the samples submitted in January to March, April to June, July to September and October to December were 22.9, 28.3, 24.5 and $24.3 \%$, respectively.

\section{Categories and definitions}

Dog ages were calculated in years that have passed since their time of birth. Dogs classed as 0 years old were puppies between 5 and 11 months old. Dogs were categorized into four BCS groups; thin dogs (15 records) were included in the underweight dog group. Additionally, dogs were classified into two sex groups (male dogs or female dogs) and also two neuter status groups (intact dogs or neutered dogs). Preliminary analysis identified two breeds, Toy Poodles and Miniature Schnauzers, as having the highest percentages of dogs with $\mathrm{DM}$; they also had relatively large sample size (450 dogs or more). In contrast, Shetland Sheepdogs have less propensity of dog DM [1], and they also had a relatively large sample size in our study (over 500 dogs). Therefore, these three breeds, Toy Poodles, Miniature Schnauzers and Shetland Sheepdogs, were used as breed groups in our study, along with a fourth group called Other breeds that comprised all the remaining 3,611 dogs. This simple grouping is done to test the hypothesis that dog breed affects dogs having DM.

\section{Statistical analysis}

Two-level analysis was applied, using a clinic at level 2 and an individual dog at level 1, to take account of the hierarchical structure of the individual dogs within a clinic. This design was used to account for the fact that an individual dog received a health check and possible treatments at an individual clinic, and that the clinic represents information similar for its average clients but not collected otherwise; such as a dog's approximate location, the dog owner's social status and the specific health guidance taken by a clinc. A two-level mixed-effects logistic regression model using the GLIMMIX procedure with logit link function was fit to the data to determine characteristics for DM in dogs. The GLIMMIX procedure with the ILINK (Inverse link function) was used to convert the logit to a probability [10]. Pairwise multiple comparisons were performed using the Tukey-Kramer test [11]. Based on an assumption of normality, figures were also generated from the estimates in the final model. All statistical analyses were performed using SAS software (SAS Int. Inc., Cary, U.S.A.).

Age, sex, neuter status, BCS group and breed group were included in the model as possible characteristics (explanatory variables). Quadratic expressions of age and all possible twoway interactions between the explanatory variables were also examined, and then removed from the model if they were not significant. A significance level of $a=0.05$ was applied. The sampled year was taken as a block in the model. Additionally, the model included the clinic as a random intercept. The adequacy of the model assumptions was evaluated by visual inspection of normal probability plots [10]. To assess the variations in the probability of dogs having DM that could be explained by the clinic, intraclass correlation coefficients (ICC) were calculated by the ICC equation in Dohoo et al. [12] :

$$
\rho=\frac{\sigma_{\text {Clinic }}^{2}}{\sigma_{\text {Clinic }}^{2}+\left(\pi^{2} / 3\right)}
$$

where $\rho$ represents the intraclass correlation coefficient, $\sigma_{\text {Clinic }}^{2}$ is the between-clinic variance and $\pi^{2} / 3$ is the assumed variance at the individual dog level.

Finally it was assessed which breeds in the studied population had the highest propensity for DM, and whether the proportion of dogs with DM differed between breeds. 
Only breeds with at least 60 dogs in the same population were selected, resulting in 10 breeds that were examined. A chi-squared test was also used to examine whether the percentage of DM dogs differed between the 10 breeds with the highest proportions of DM dogs (only breeds with sample size at least 60 dogs).

\section{Results}

The mean age in all the studied dogs was $8.6 \pm 0.05$ years old ranging from 0 to 17 years. Of 2,372 male dogs and 2,585 female dogs, $62.2 \%$ and $74.2 \%$ were neutered, respectively. Relative frequencies (\%) of BCS $1,2,3,4$ and 5 were $0.3,4.2$, $41.4,39.7$ and $14.4 \%$, respectively (Table 1$)$. Prevalence $( \pm$ SEM) of dogs having DM was $3.6 \pm 0.01 \%$ in this studied population.

Table 2 shows the final model for dogs having DM. Dogs having DM was associated with age, BCS and breed groups $(P<0.05)$, whereas was not associated with $(P=0.41)$ and neuter status $(P=0.68)$. Increased age was non-linearly associated with a higher probability of dogs having $\mathrm{DM}(\mathrm{P}<0.05)$, with the probability peaking at 9 years of age, and then declining (Figure 1). The odds ratio of dogs having DM was 5.6 for underweight and thin dogs compared with ideal dogs $(P<0.05)$.

Table 1. Relative frequency of 5,343 dogs categorized on the basis of body condition score groups.

\begin{tabular}{lll}
\hline Measurements & $\mathbf{N}$ & $\mathbf{\%}$ \\
\hline Body condition score group & -- & -- \\
1: Thin & 15 & 0.3 \\
2: Underweight & 207 & 4.2 \\
3: Ideal & 2,052 & 41.4 \\
4: Overweight & 1,967 & 39.7 \\
5: Obese & 714 & 14.4 \\
\hline
\end{tabular}

*The remaining records $(5,343-\mathrm{N})$ were treated as missing values.

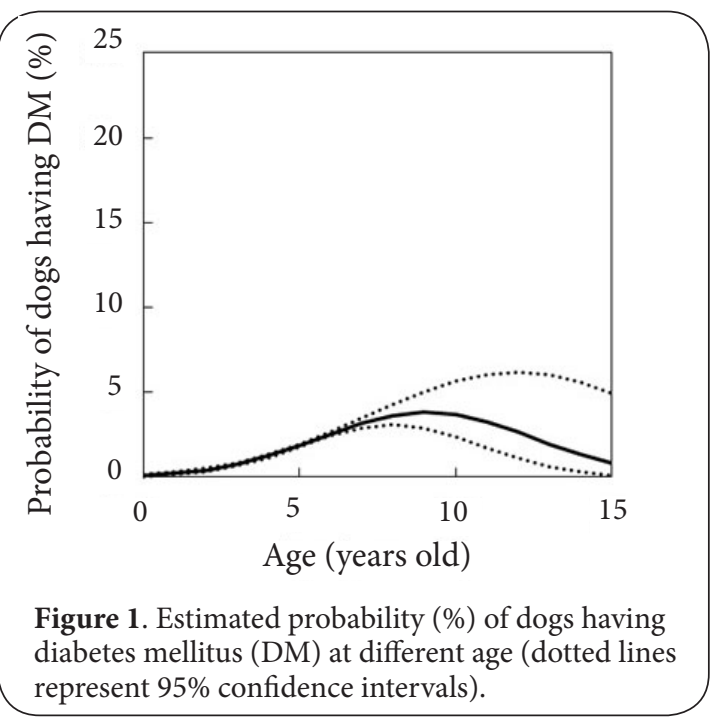

Table 2. Estimates of fixed effects and random effect variance included the final binary logistic regression model (including breed groups) of the probability of a dog having diabetes mellitus (DM).

\begin{tabular}{|c|c|c|}
\hline Fixed effects and variance & Estimate $( \pm \mathrm{SE})$ & P-value \\
\hline Intercept & $-3.378(0.293)$ & $<0.01$ \\
\hline Age & $0.092(0.104)$ & 0.11 \\
\hline Age squared & $-0.042(0.010)$ & $<0.01$ \\
\hline Body condition score group & -- & $<0.01$ \\
\hline Obese dogs & 0 & -- \\
\hline Overweight dogs & $-0.316(0.333)$ & -- \\
\hline Ideal dogs & $0.044(0.303)$ & -- \\
\hline Underweight and thin dogs & $1.816(0.372)$ & -- \\
\hline Breed group & -- & $<0.01$ \\
\hline Toy Poodles & $1.218(0.274)$ & -- \\
\hline Miniature Schnauzers & $0.580(0.248)$ & -- \\
\hline Shetland Sheepdogs & $-2.719(1.164)$ & -- \\
\hline Others & 0 & -- \\
\hline AgexBody condition score group & -- & 0.01 \\
\hline AgexObese dogs & 0 & -- \\
\hline AgexOverweight dogs & $0.093(0.124)$ & -- \\
\hline AgexIdeal dogs & $-0.052(0.118)$ & -- \\
\hline AgexUnderweight and thin dogs & $-0.258(0.131)$ & -- \\
\hline AgexBreed group & -- & 0.02 \\
\hline AgexToy Poodles & $0.288(0.092)$ & -- \\
\hline AgexMiniature Schnauzers & $0.125(0.096)$ & -- \\
\hline AgexShetland Sheepdogs & $0.150(0.417)$ & -- \\
\hline AgexOthers & 0 & -- \\
\hline Clinic variance & 0.46 & -- \\
\hline Intraclass correlation coefficient, \% & 12.2 & -- \\
\hline
\end{tabular}

*Year effect was not shown because it was not relevant to the study objectives.

Additionally, underweight and thin dogs had 9.4-10.1\% higher probability of having DM than dogs in the other BCS groups ( $P<0.05$; Table 3$)$. The odds ratios of Toy Poodles and Miniature Schnauzers were 3.1 and 1.6, respectively $(P<0.05)$ compared to Other breeds. Also, Toy Poodles and Miniature Schnauzers had $4.9-9.3 \%$ higher probabilities of having DM than Shetland Sheepdogs $(\mathrm{P}<0.05)$.

There were two two-way interactions between the dog age and $B C S(P=0.01)$ or breed groups $(P=0.02)$, respectively, for dogs having DM (Table 2). In underweight and thin dogs, the probability of dogs having DM increased from $4.5 \%$ to a peak of $21.2 \%$ as dog age rose from 1 to 7 years old $(P<0.05$; Figure 2), whereas in obese and overweight dogs the probabilities increased from 0.14 to $2.3 \%$, and from 0.07 to $2.2 \%$, respectively, over the same age range. There was only a low overall probability of dogs of ideal weight having DM, but the probability still rose from $0.2 \%$ at 1 years old to a peak of $4.3 \%$ at 9 years old.

Figure 3 shows that the probability of Toy Poodles and Miniature Schnauzers having DM increased non-linearly from 
Usui et al. Veterinary Medicine and Animal Sciences 2015,

http://www.hoajonline.com/journals/pdf/2054-3425-3-5.pdf

doi: $10.7243 / 2054-3425-3-5$

Table 3. Comparisons between explanatory variables (risk factors) for the probability of dogs having diabetes mellitus (DM) and the odds ratios from the final model for the DM.

\begin{tabular}{|c|c|c|c|c|}
\hline \multirow[t]{2}{*}{ Risk factors ${ }^{*}$} & \multicolumn{2}{|c|}{$\begin{array}{l}\text { Probability of dogs } \\
\text { having DM, \% }\end{array}$} & \multirow{2}{*}{$\begin{array}{l}\text { Odds ratio } \\
\text { (95\% confidence } \\
\text { interval) }\end{array}$} & \multirow[t]{2}{*}{ P-value } \\
\hline & $\mathbf{N}^{* *}$ & Mean $\pm \mathrm{SE}^{\dagger}$ & & \\
\hline \multicolumn{5}{|c|}{ Body condition score group } \\
\hline Obese dogs & 714 & $1.6 \pm 0.50^{\mathrm{b}}$ & $0.7(0.4-1.2)$ & 0.19 \\
\hline Overweight dogs & 1,967 & $1.7 \pm 0.43^{\mathrm{b}}$ & $0.7(0.5-1.1)$ & 0.17 \\
\hline Ideal dogs & 2,052 & $2.3 \pm 0.53^{b}$ & Reference & -- \\
\hline $\begin{array}{l}\text { Underweight } \\
\text { and thin dogs }\end{array}$ & 222 & $11.7 \pm 3.11^{\mathrm{a}}$ & $5.6(3.3-9.8)$ & $<0.01$ \\
\hline \multicolumn{5}{|l|}{ Breed group ${ }^{\S}$} \\
\hline Toy Poodles & 453 & $9.8 \pm 2.10^{\mathrm{a}}$ & $3.1(1.9-5.0)$ & $<0.01$ \\
\hline $\begin{array}{l}\text { Miniature } \\
\text { Schnauzers }\end{array}$ & 766 & $5.4 \pm 1.15^{\mathrm{b}}$ & $1.6(1.0-2.6)$ & 0.04 \\
\hline $\begin{array}{l}\text { Shetland Sheep- } \\
\text { dogs }\end{array}$ & 513 & $0.5 \pm 0.38^{\mathrm{d}}$ & $0.1(0.0-0.7)$ & 0.01 \\
\hline Others & 3,611 & $3.4 \pm 0.46^{c}$ & Reference & -- \\
\hline
\end{tabular}

${ }^{\mathrm{a}-\mathrm{d}}$ Means within a column with different letters differ $(\mathrm{P}<0.05)$.

*Year effect was not shown because it was not a research purpose.

${ }^{*}$ The remaining records $(5,343-\mathrm{N})$ were treated as missing values.

${ }^{\dagger}$ Mean and SE were estimated by a mixed-effects multivariable model.

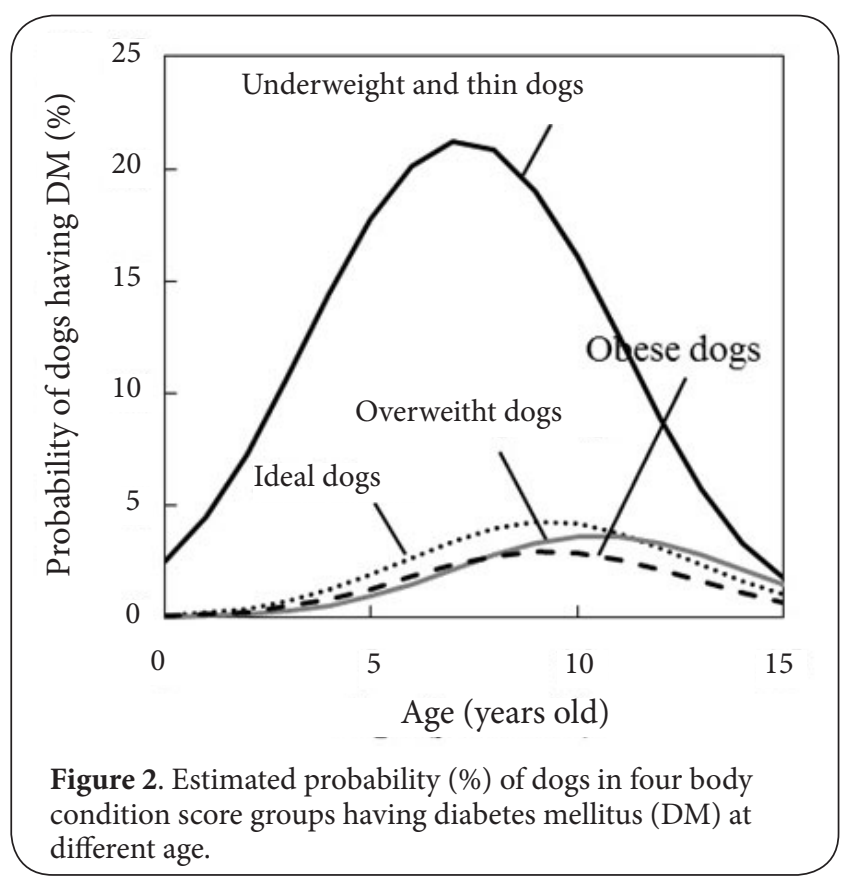

2.2 to $18.6 \%$ and from 2.0 to $6.6 \%$, respectively, as dog age rose from 5 to 12 years old $(P<0.05)$, whereas over the same age range DM in Shetland Sheepdogs increased non-linearly from 0.2 to only $0.6 \%$.

Toy Poodles had the highest percentage of dogs having DM out of 102 breeds (Table 4). A chi-squared test also showed

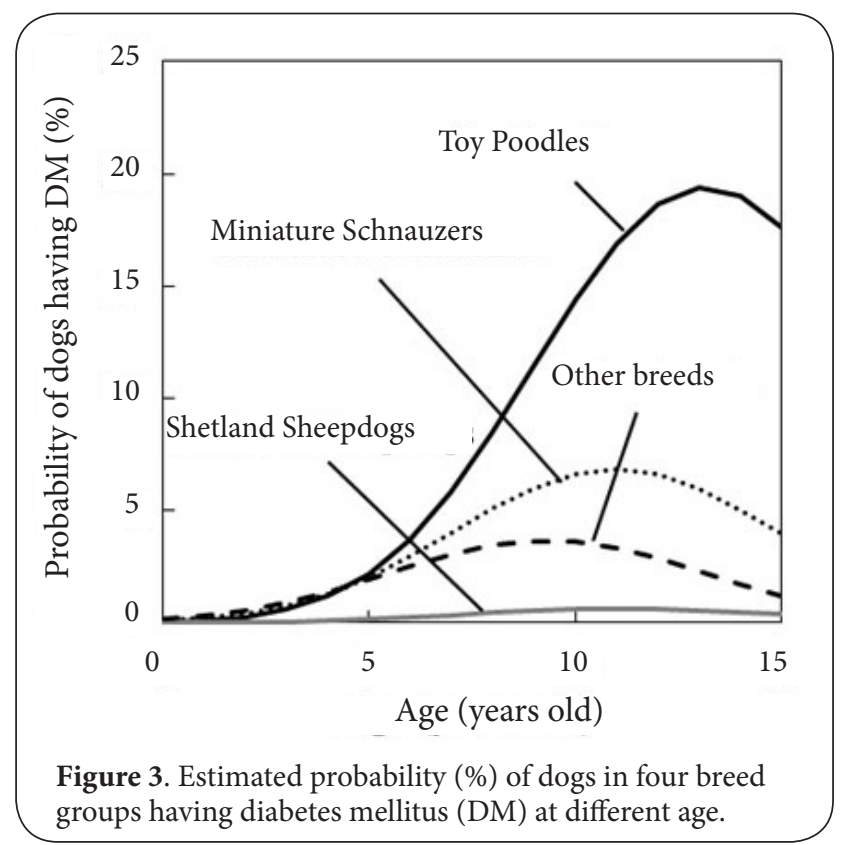

that the percentages of DM dogs differed between the 10 selected breeds $(P<0.05)$. Finally, with regard to the ICC, the clinic effect explained $11.0-13.4 \%$ of the total variation for the probability of dogs having DM.

\section{Discussion}

The present study quantified the associations between increased age and the probability of dogs having DM, and showed that the probability peaked at middle age. Also, in our study most dogs (65.1\%) were found to have had DM between 5 and 11 years of age. These results confirm findings from a previous study [4], indicating that DM develops over months or years through several pathophysiological stages before dogs are diagnosed as DM dogs.

Our study also showed that dogs evaluated as underweight or thin have a higher odds ratio of being diagnosed for DM as compared to dogs in the other BCS groups. Weight loss is one of the clinical signs for DM, and therefore DM dogs are likely to become underweight and thin [4]. The results in the present study and those in a previous study [4] imply that the owners are more likely to recognize that their dogs have a disease problem due to the dogs being underweight, thin or suffering weight loss. In our study obesity was not associated with $\mathrm{DM}$, but obesity may be a factor because it is associated with an increased risk of pancreatitis and glucose intolerance, both of which are a common cause of DM $[3,4]$.

There was also an interaction between age and breed, which indicates that the probability of dogs having DM in middle age or older varies greatly between breed groups. The higher likelihood of Toy Poodles and Miniature Schnauzers having DM is consistent with previous studies $[1,13]$. Overall, the differences in the percentages of DM dogs between 
Table 4. Top 10 breeds with the highest percentages of dogs having diabetes mellitus (DM) out of 102 breeds studied ${ }^{*}$.

\begin{tabular}{llllll}
\hline Rank & Breed & Body size & $\begin{array}{l}\text { Number } \\
\text { of dogs }\end{array}$ & $\begin{array}{l}\text { Number } \\
\text { DM dogs }\end{array}$ & $\begin{array}{l}\text { Percentage of } \\
\text { DM dogs, \% }\end{array}$ \\
\hline 1 & Toy Poodle & Toy & 453 & 37 & 8.2 \\
2 & Miniature Schnauzer & Small & 766 & 48 & 6.3 \\
3 & Pomeranian & Toy & 237 & 13 & 5.5 \\
4 & Maltese & Toy & 187 & 10 & 5.3 \\
5 & Labrador Retriever & Large & 61 & 3 & 4.9 \\
6 & Yorkshire Terrier & Toy & 250 & 12 & 4.8 \\
7 & Mongrel & Unknown & 372 & 17 & 4.6 \\
8 & Welsh Corgi & Medium & 100 & 4 & 4.0 \\
9 & Beagle & Medium & 88 & 2 & 2.3 \\
10 & Shiba Inu & Small & 146 & 3 & 2.1 \\
\hline
\end{tabular}

*The Table includes only 10 breeds with more than 60 dogs.

the top 10 breeds (Table 3 ) indicate that breeds have an important predictor for dogs having DM. Such differences in breed susceptibility to DM are associated with multiple genes, which increase the risk of DM, and differences in the haplotype between breeds $[\mathbf{2}, \mathbf{1 4}]$.

The lack of any effects of sex or neuter status on the probability of dog DM may be explained by the fact that our data included many neutered dogs. Approximately $74 \%$ of the female dogs $(1,919 / 2,585$ female dogs) were neutered, and it is likely that many or most of these dogs would have been neutered at an early age, although the records did not include the timing of neutering. Previous studies have shown that female dogs were more likely to have DM compared to male dogs [1], and that intact female dogs had a higher percentage of DM than neutered female dogs [2]. Neutering reduces synthesis of progesterone [2], and this is likely to reduce the risk of DM in neutered dogs [4]. Therefore, neutering at an early stage is recommended in the U.K. [2] and also it has been shown that neuter status is not a factor for DM [1].

\section{Conclusions}

Characteristics of dogs having DM were high age, low BCS and specific breeds. This finding could help veterinarians to identify subclinical DM dogs, by taking these characteristics into account. Our study also shows that there are some difference between clinics in terms of DM dogs, as indicated by the ICCs of $11.0-13.4 \%$ for clinic variance. This suggests that some clinic related factors could be related to dog DM, such as clinic location, a neighborhood and social economic status of the average owner in that area and veterinarians' guidance for dogs.

Finally, it should be noted that there are some limitations to this present study. Dogs were not randomly selected because it was a cross-sectional study to examine possible risk factors using veterinarian-submitted samples. The proportion of DM
(3.6\%) in our study was relatively high. It is possible that samples from clinics with a high prevalence of DM dogs are overpresented. Also, the veterinarians did not record separately the type of diabetes that each dog had. Additionally, there is still possibility that we have had samples from certain clinics which may be highly related to high or low risk DM breeds. Furthermore, neither the dogs' rearing environments nor their nutrition were taken into account in the analyses. The level of agreement between the BCS evaluations conducted by the participating veterinarians was not evaluated. However, our statistical models included the clinic as a random effect in order to adjust some clinic or veterinarian effects. Even with such limitations, this research provides valuable information for veterinarians about the characteristics related to dog DM.

\section{Competing interests}

The authors declare that they have no competing interests.

Authors' contributions

\begin{tabular}{|l|c|c|c|}
\hline Authors' contributions & SU & HY & YK \\
\hline Research concept and design & $\checkmark$ & -- & $\checkmark$ \\
\hline Collection and/or assembly of data & $\checkmark$ & $\checkmark$ & -- \\
\hline Data analysis and interpretation & $\checkmark$ & -- & $\checkmark$ \\
\hline Writing the article & $\checkmark$ & -- & $\checkmark$ \\
\hline Critical revision of the article & -- & $\checkmark$ & $\checkmark$ \\
\hline Final approval of article & $\checkmark$ & $\checkmark$ & $\checkmark$ \\
\hline Statistical analysis & $\checkmark$ & -- & $\checkmark$ \\
\hline
\end{tabular}

\section{Acknowledgement}

The authors gratefully thank the cooperating veterinarians for providing their data for use in the present study. We also thank Dr. I. McTaggart for his critical review of this manuscript.

\section{Publication history}

Editor: Olaf Berke, University of Guelph, Canada. Received: 20-Aug-2015 Final Revised: 28-Sep-2015 Accepted: 06-Oct-2015 Published: 15-Oct-2015 
Usui et al. Veterinary Medicine and Animal Sciences 2015,

\section{References}

1. Guptill $L$, Glickman $L$ and Glickman N. Time trends and risk factors for diabetes mellitus in dogs: analysis of veterinary medical data base records (1970-1999). Vet J. 2003; 165:240-7. | Article | PubMed

2. Catchpole B, Ristic JM, Fleeman LM and Davison LJ. Canine diabetes mellitus: can old dogs teach us new tricks? Diabetologia. 2005; 48:194856. | Article | PubMed

3. Mattin M, O'Neill D, Church D, McGreevy PD, Thomson PC and Brodbelt $D$. An epidemiological study of diabetes mellitus in dogs attending first opinion practice in the UK. Vet Rec. 2014; 174:349. | Article | PubMed

4. Fleeman L and Rand J. Canine Diabetes Mellitus. In Rand J (Ed), Clinical Endocrinology of Companion Animals. Wiley-Blackwell, Ames. 2013; 143-168.

5. Hess RS, Saunders HM, Van Winkle TJ and Ward CR. Concurrent disorders in dogs with diabetes mellitus: 221 cases (1993-1998). J Am Vet Med Assoc. 2000; 217:1166-73. | Article | PubMed

6. Edney AT and Smith PM. Study of obesity in dogs visiting veterinary practices in the United Kingdom. Vet Rec. 1986; 118:391-6. | Article | PubMed

7. Laflamme D. P. Development and validation of a body condition score system for dogs. Canine Practice. 1997; 22:10-15. | Article

8. JKC (Japan Kennel Club). Popular dog breeds in Japan. 2009.

9. MAFF (Ministry of Agriculture, Forestry and Fisheries). Statistics 2013 in the number of animal hospitals in Japan.

10. Littell RC, Milliken GA, Stroup WW, Wolfinger RD and Schabenberger O. SAS System for Mixed Models. 2nd edn. SAS Inst. Inc., Cary. 2006; 525-566.

11. SAS/STAT User Guide. The GLIMMIX Procedure. SAS Institute, Cary. 2013; 3080-3209. | Pdf

12. Dohoo IR, Martin SW and Stryhn H. Veterinary Epidemiologic Research. 2nd edn. VER Inc., Charlottetown. 2009; 579-606.

13. Hess RS, Kass PH and Van Winkle TJ. Association between diabetes mellitus, hypothyroidism or hyperadrenocorticism, and atherosclerosis in dogs. J Vet Intern Med. 2003; 17:489-94. | Article | PubMed

14. Short AD, Saleh NM, Catchpole B, Kennedy LJ, Barnes A, Jones CA, Fretwell $\mathrm{N}$ and Ollier WE. CTLA4 promoter polymorphisms are associated with canine diabetes mellitus. Tissue Antigens. 2010; 75:24252. | Article | PubMed

\section{Citation:}

Usui S, Yasuda $\mathrm{H}$ and Koketsu Y. Characteristics of dogs having diabetes mellitus; analysis of data from private Japanese veterinary clinics. Vet Med Anim Sci. 2015; 3:5. http://dx.doi.org/10.7243/2054-3425-3-5 